\title{
Remote ischemic preconditioning versus standard myocardial protection in cardiac surgery: ten years of clinical trials. A systematic review and meta-analysis
}

\author{
Jakub Marczak ${ }^{1,3}$, Tomasz Bańkowski², Tomasz Płonek ${ }^{3}$, Marta Negrusz-Kawecka², \\ Wojciech Kustrzycki ${ }^{3}$ \\ ${ }^{1}$ Lower Silesian Center for Heart Diseases, Department of Surgery, Nowa Sól, Poland \\ 2Department of Cardiology, Wroclaw Medical University, Wroclaw, Poland \\ ${ }^{3}$ Department of Cardiac Surgery, Wroclaw Medical University, Wroclaw, Poland
}

Kardiochirurgia i Torakochirurgia Polska 2013; 10 (3): 268-278

\section{Summary}

Objectives: To systematically review and assess the existing evidence for the applicability of remote ischemic preconditioning (rIPC) in cardiac surgery.

Material and methods: Major biomedical databases: Medline, Cochrane, etc. were searched. All randomized controlled trials (RCTs) comparing rIPC and standard myocardial protection in patients submitted to cardiac surgery were included if they reported at least one of the outcomes of interest: myocardial injury markers, postoperative inotropic support, or length of ICU stay.

Results: 991 patients were included in the analysis. rIPC was shown to reduce myocardial injury markers postoperatively by -0.63 SMD [ -0.99 to -0.28$]$ and postoperative inotropic requirement by -0.40 SMD $[-0.66$ to -0.16$]$ in the adult patients submitted to cardiac surgery. There has been significant, yet correctable, heterogeneity of the primary outcome of interest, and the available RCTs were small sample studies.

Conclusions: This meta-analysis provides evidence confirming that rIPC has potential benefits with regard to myocardial protection.

Key words: meta-analysis, remote ischemic preconditioning, myocardial protection, cardiac surgery.

\section{Introduction}

A significant amount of research has been performed to provide a closer look at the ischemia-reperfusion phenom-

\section{Streszczenie}

Celem niniejszej metaanalizy jest systematyczny przegląd dowodów obrazujących wpływ odległego hartowania niedokrwiennego (rIPC) na mięsień serca pacjentów poddanych operacjom kardiochirurgicznym.

Materiały i metody: Przeglądu literatury dokonano na podstawie pełnotekstowych prac publikowanych $w$ bazach danych Medline, Cochrane itp. Włączono badania kontrolowane z randomizacją, porównujące rIPC oraz standardową ochronę mięśnia serca u pacjentów poddanych zabiegom kardiochirurgicznym, jeżeli raportowały co najmniej jeden z wyników: markery uszkodzenia mięśnia sercowego, pooperacyjne zapotrzebowanie na leki wykazujące dodatni efekt inotropowy, czas hospitalizacji na oddziale intensywnej terapii.

Wyniki: Do metaanalizy włączono 991 pacjentów. W porównywanych badaniach rIPC zmniejszało istotnie statystycznie ilość uwalnianych pooperacyjnie markerów martwicy mięśnia sercowego o-0,63 SMD $[-0,99$ do -0,28] oraz pooperacyjne zapotrzebowanie na leki inotropowe o-0,40 SMD [-0,66 do -0,16] w populacji osób dorosłych. Badanie było obarczone korygowalnym statystycznie współczynnikiem heterogeniczności. Publikowane dane przygotowano na podstawie badań pilotażowych.

Wnioski: Poniższa metaanaliza wczesnych dowodów klinicznych potwierdza istnienie potencjalnych korzyści ze stosowania rIPC w celu zmniejszenia okołooperacyjnego uszkodzenia mięśnia sercowego.

Słowa kluczowe: metaanaliza, odległe hartowanie niedokrwienne, kardioprotekcja, kardiochirurgia.

enon and at interventions that could overcome the associated injury. There is no other way to salvage the ischemic myocardium than to restore and maintain blood flow to

Address for correspondence: lek. Jakub Sebastian Marczak, Lower Silesian Center for Heart Diseases, Chałubińskiego 7 Str., 67-100 Nowa Sól, Poland, phone: +48 8837745 66, fax: +48 6838821 91, e-mail: marczak@space.pl 
the hypoxic tissue [1]. This well-known therapeutic paradigm has unfortunately been associated with additional injury mediated by free radicals generated during the transition from anaerobic to aerobic metabolism upon the restoration of blood flow [2]. Several mechanisms (such as intracellular calcium oscillation, opening of mitochondrial transition pores, or simply oxidative stress) are thought to play a role in the additional necrosis or myocardial stunning associated with reperfusion. Not surprisingly, during the 1990s, much scientific effort was devoted to these physiological phenomena, giving rise to various therapeutic approaches [3]. Inducing non-lethal and brief ischemia before the period of prolonged ischemia has been considered as a tool for increasing the heart's resistance to ischemiareperfusion (I/R) injury, as tested in pre-clinical studies and in human volunteers $[4,5]$. Subsequently, preconditioning the heart with ischemia was shown to maintain its cardioprotective abilities even if the non-lethal ischemic stimulus was applied not directly to the targeted tissue, but to any distant site of the organism - hence the idea of remote ischemic preconditioning (rIPC) [6]. In cardiac surgery, where the timing of global ischemia and reperfusion periods is predictable, the application of rIPC seemed a perfect solution [7]. It has been ten years since the first application of rIPC in CABG patients, and, so far, approximately one thousand patients have been submitted to cardiac surgery with or without rIPC. Over the decade, leading cardiovascular researchers have gone from great excitation to dramatic disappointment with the clinical application of $\operatorname{rIPC}[8,9]$. The frustration could not be overcome by the recent metaanalyses and narrative reviews, due to their low quality or lack of objective quantitative measures of the effect, respectively [10-13]. This review and meta-analysis provides the most up-to-date and in-depth quantitative analysis of rIPC in adult and pediatric cardiac surgery.

This generic study and literature review was performed to critically appraise the evidence for the effect of rIPC on myocardial protection against I/R injury. This was achieved by means of a quantitative analysis comparing the release of postoperative myocardial injury markers in the patients who received the intervention and those who did not. Inotropic support requirement was analyzed, as it correlates with the extent of myocardial stunning following heart surgery, a core effect of I/R. ICU length of stay was also assessed. Heterogeneity assessment and sensitivity analysis were undertaken in the case of discrepancies between contrary results.

\section{Material and methods}

\section{Literature search}

Major contemporary biomedical databases were searched in order to make the review as comprehensive as possible. The databases included: Medline/PubMed (1950August 2011), Google Scholar (1992 - August 2011), Web of Knowledge (1945 - August 2011), CINAHL (1980 - August 2011), EMBASE (1980 - August 2011), Cochrane Library/ Central Register of Controlled Trials (CENTRAL). The pri- mary search employed the following key terms: "remote is chemic preconditioning" and/or "remote ischemic pre conditioning" and/or "remote ischemia" and "cardiac surgery". The 'related articles' function was used in order to ensure the broadest possible data retrieval. All relevant medical literature, regardless of the language of reporting and type of publication, were retrieved by the aforementioned search. Titles and abstracts of these publications were checked against predefined criteria for eligibility. These were as follows: (1) RCT design, (2) trials comparing rIPC versus controls (placebo with standard myocardial protection), (3) trials reporting at least one outcome of interest, (4) trials not duplicating results obtained from one study group, (5) trials published in peer-reviewed journals. These criteria excluded 'grey literature' and papers that duplicated findings derived from one study group; when the latter was the issue, the most representative report was included for further assessment. The search strategy is presented in Fig. 1.

\section{Data extraction}

A pre-designed form was used in order to provide the medium for data extraction. Two independent searches and data extractions were made by two different authors: JM and TP. There was unequivocal agreement between

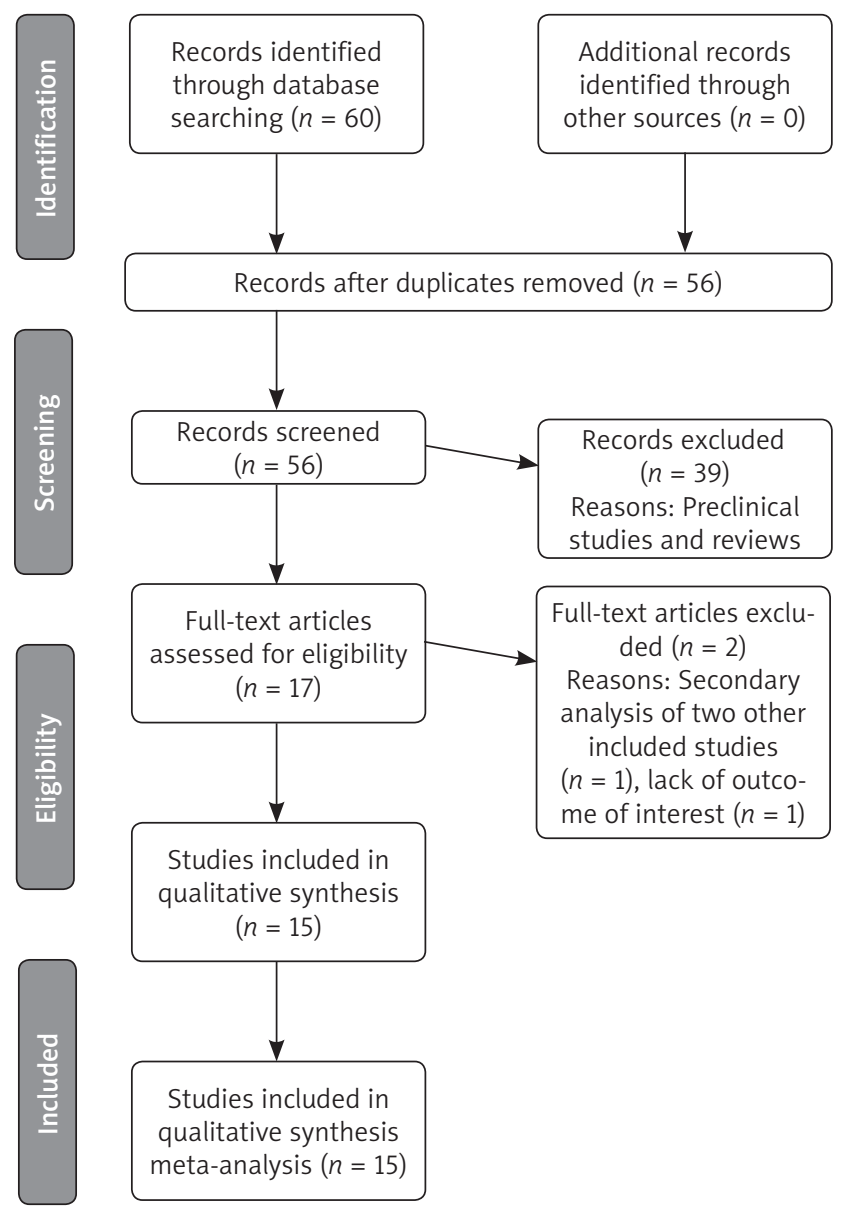

Fig. 1. PRISMA flow diagram 
the authors as to which papers should be included, given the proposed criteria. Both authors used the same form to assess the three major points of systematic review: eligibility criteria, outcomes of interest, and the risk of bias associated with individual papers using the Cochrane Collaboration criteria and the Jadad score [14]. Any disagreement during the process was resolved by consensus. If data were missing from a report, the following algorithm was applied: the authors of the report were contacted and requested to provide the missing data. If that did not suffice, the missing data were assessed and, if feasible, computed from other data available in the paper (missing variance or inappropriate variance format). Only one case required the application of this second step: the missing variance value was replaced by the result of variance calculation using the algorithm for interquartile range assessment of variance [15].

\section{Definitions of outcomes of interest}

Our study had one primary and two secondary outcomes of interest. The primary outcome was the release of myocardial injury markers following a cardiac procedure, while the secondary outcomes were inotropic support requirement and ICU length of stay. The data were insufficient to construct an analysis based on hard clinical endpoints, such as peri-procedural myocardial infarction (PMI) or in-hospital mortality; therefore, the review was based on surrogate outcomes.

\section{Statistical analysis}

The report was written in accordance with the PRISMA Statement (Preferred Reporting Items for Systematic Reviews and Meta-Analyses) and the Cochrane Collaboration recommendations $[16,17]$. The meta-analysis was performed using Cochrane Collaboration Software - Review Manager [18]. The magnitude and the direction of effects computed from continuous data were presented as the standardized mean difference (SMD). When the out-

Tab. I. Methodological quality assessment (Jadad score)

Generation of allocation sequence:

2: computer-generated random numbers

1: not described

Allocation concealment:

3: central randomization

2: sealed envelopes or similar

1: not described or inadequate

\section{Investigator blindness:}

2: identical placebo tablets or similar

1: inadequate or not described

0 : no double-blinding

Description of withdrawals and drop-outs:

1: numbers and reasons are described

0 : numbers and reasons are not described

Efficacy of randomization:

2: pretreatment variables in tabular form

1: balance of pretreatment variables mentioned but not in tabular form

0 : no information reported come was reported consistently on the same scale, the result was presented as mean difference (MD). All outcomes are presented with appropriate $95 \%$ confidence intervals. Statistical significance was assumed at $p<0.05$.

\section{Assessment of data validity and heterogeneity}

Validity, heterogeneity, and the risk of bias were approached in both a graphical and a quantitative manner. A random effect model was preferred when significant heterogeneity was the issue, as it allows for a larger margin of variance between individual studies. A fixed effect model was applied when heterogeneity was low $\left(1^{2}<25 \%\right)$. Heterogeneity was assessed with standard $\chi^{2}$ calculation and the $\mathrm{I}^{2}$ statistic. The $\mathrm{I}^{2}$ statistic allows for the assessment of the observed variation between studies, which can be assigned as having a low (<25\%), moderate (25-75\%), or high ( $>75 \%$ ) degree of heterogeneity [19].

The estimation of publication bias and the risk of bias was conducted in compliance with the Cochrane Collaboration guidelines [17]. Publication bias was evaluated by funnel plot asymmetry assessment. Studies lying outside of the $95 \% \mathrm{Cl}$ were considered causative of asymmetry. The risk of bias was assessed in a standard Cochrane manner with regard to six domains representing different items which might be responsible for systematic deviation from true results, i.e. selection, performance, detection, attrition, reporting, and other bias. Additionally, the Jadad score was calculated for every paper to provide more data concerning the risk of bias associated with individual papers (Table I). Sensitivity analysis was conducted in order to compare the fixed effect and random effect models. Both models were applied in order to assess the differences in their possible impact on the meta-analytical results. Subgroup analysis was managed by analyzing adult CABG trials, adult valve trials, and pediatric cardiac trials separately. Individual analysis was also performed on studies of high or low risk of bias, studies using different sites of RIPC application (lower or upper extremity), and studies including both diabetic and non-diabetic patients.

\section{Results}

\section{Literature search and study characteristics}

Using the reported search, sixty papers were found, of which fifteen were in agreement with the inclusion criteria. References of these articles were cross-searched to reveal any documents missing from the review. Finally, fifteen papers were found to present the best available evidence. These studies pertained to: adult coronary bypass grafting surgery with or without AVR [9, 12-27], adult valvular surgery [28-30], and pediatric cardiac surgery [31-33]. All the available papers were designed as prospective randomized controlled trials. The characteristics and outcomes of the investigated studies are presented in Table II and Table III.

The meta-analysis involved a total of 991 patients, with 491 receiving rIPC and 500 in the control group. The mean 
Tab. II. Characteristics of investigated trials

\begin{tabular}{|c|c|c|c|c|c|c|}
\hline Study/year & $\begin{array}{l}\text { Participants } \\
\text { RIPC/Controls }\end{array}$ & $\begin{array}{l}\text { Gender-male } \\
\text { RIPC/Controls }\end{array}$ & $\begin{array}{c}\text { Age } \\
\text { RIPC/Controls }\end{array}$ & $\begin{array}{l}\text { Type of surgical } \\
\text { procedure }\end{array}$ & $\begin{array}{l}\text { Means of } \\
\text { reporting } \\
\text { outcomes }\end{array}$ & $\begin{array}{l}\text { RIPC protocol } \\
\text { Ischemia/Reperfusion } \\
\text { (min) }\end{array}$ \\
\hline Cheung, 2006 [31] & $17 / 20$ & - & $\begin{array}{c}0.9( \pm 0.9) / 2.2( \pm 3.4) \\
\text { years }( \pm S D)\end{array}$ & $\begin{array}{c}\text { VSD mainly ASD } \\
\text { and } \\
\text { TGA or ToF }\end{array}$ & Troponin I & $\begin{array}{l}5 / 5 \text { min, } 4 \text { cycles } \\
\text { (lower extremity) }\end{array}$ \\
\hline Zhou, 2010 [32] & $30 / 30$ & $16 / 18$ & $\begin{array}{c}160.83( \pm 58.39) / 154.13 \\
( \pm 55.82), \text { days }( \pm S D)\end{array}$ & $\begin{array}{l}\text { VSD closure } \\
\text { (elective) }\end{array}$ & $\begin{array}{l}\text { Troponin I, CK } \\
\text { MB and LDH }\end{array}$ & $\begin{array}{l}5 / 5 \text { min, } 2 \text { cycles } \\
\text { (upper extremity) }\end{array}$ \\
\hline Wagner, 2010 [22] & $32 / 34$ & $24 / 23$ & 67 years/71 years & $\begin{array}{l}\text { CABG } \pm \text { AVR } \\
\text { (elective) }\end{array}$ & $\begin{array}{l}\text { Troponin I } \\
C_{\max } \text { within } \\
24 \text { hours }\end{array}$ & $\begin{array}{l}5 / 5 \text { min, } 3 \text { cycles } \\
\text { (upper extremity) }\end{array}$ \\
\hline Choi, 2011 [29] & $38 / 38$ & $15 / 15$ & $\begin{array}{c}57( \pm 12) / 60( \pm 13) \\
\text { years }( \pm S D)\end{array}$ & $\begin{array}{c}\text { AVR/MVR/DVR } \\
\text { and Bentall proce- } \\
\text { dures } \pm \text { CABG }\end{array}$ & $\begin{array}{l}\text { CK-MB at } 12^{\text {th }} \\
\text { and } 24^{\text {th }} \text { hour }\end{array}$ & $\begin{array}{l}\text { 10/10 min, } 3 \text { cycles } \\
\text { (lower extremity) }\end{array}$ \\
\hline Li, 2010 [28] & $26 / 27$ & 9/13 & $\begin{array}{c}45.8( \pm 11.2) / 42.3 \\
( \pm 10.6), \text { years }( \pm S D)\end{array}$ & $\begin{array}{l}\text { AVR/MVR and DVR } \\
\text { (elective) }\end{array}$ & $\begin{array}{l}\text { Troponin I } \\
\text { AUC over } \\
72 \text { hours }\end{array}$ & $\begin{array}{l}5 / 5 \text { min, } 3 \text { cycles } \\
\text { (lower extremity) }\end{array}$ \\
\hline $\begin{array}{l}\text { Hausenloy, } \\
2007[9]\end{array}$ & $27 / 30$ & $21 / 24$ & $\begin{array}{c}67( \pm 11.8) / 67( \pm 9.4) \\
\text { years }( \pm S D)\end{array}$ & CABG (elective) & $\begin{array}{l}\text { Troponin T } \\
\text { AUC over } \\
72 \text { hours }\end{array}$ & $\begin{array}{l}5 / 5 \text { min, } 3 \text { cycles } \\
\text { (upper extremity) }\end{array}$ \\
\hline Rahman, 2010 [19] & $80 / 82$ & $72 / 71$ & 65 years/63 years & $\begin{array}{l}\text { CABG (urgent and } \\
\text { elective) }\end{array}$ & $\begin{array}{l}\text { Troponin T } \\
\text { AUC over } \\
48 \text { hours }\end{array}$ & $\begin{array}{l}5 / 5 \text { min, } 3 \text { cycles } \\
\text { (upper extremity) }\end{array}$ \\
\hline Ali, 2010 [21] & $50 / 50$ & $47 / 42$ & $\begin{array}{c}56.0( \pm 8.2) / 51.6( \pm 9.6) \\
\text { years }( \pm S D)\end{array}$ & $\begin{array}{c}\text { CABG (elective) } \\
\pm \text { CEA }\end{array}$ & $\begin{array}{l}\text { CK-MB } C_{\max } \\
\text { within } \\
48 \text { hours } \\
\end{array}$ & $\begin{array}{l}5 / 5 \text { min, } 3 \text { cycles } \\
\text { (upper extremity) }\end{array}$ \\
\hline $\begin{array}{l}\text { Thielmann, } \\
2010[25]\end{array}$ & $27 / 26$ & $23 / 22$ & $64.1 / 63.4$ years & CABG (elective) & $\begin{array}{l}\text { Troponin I } \\
C_{\max } \text { within } \\
24 \text { hours }\end{array}$ & $\begin{array}{l}5 / 5 \text { min, } 3 \text { cycles } \\
\text { (upper extremity) }\end{array}$ \\
\hline $\begin{array}{l}\text { Venugopal, } \\
2009 \text { [24] }\end{array}$ & $23 / 22$ & $19 / 19$ & $\begin{array}{c}62( \pm 9.7) / 64( \pm 9.0) \\
\text { years }( \pm S D)\end{array}$ & $\begin{array}{l}\mathrm{CABG} \pm \mathrm{AVR} \\
\text { (elective) }\end{array}$ & $\begin{array}{l}\text { Troponin I } \\
\text { AUC over } \\
72 \text { hours }\end{array}$ & $\begin{array}{l}5 / 5 \text { min, } 3 \text { cycles } \\
\text { (upper extremity) }\end{array}$ \\
\hline $\begin{array}{l}\text { Gunaydin, } \\
2000[26]\end{array}$ & $4 / 4$ & $4 / 4$ & $\begin{array}{c}62.2( \pm 6.2) / 60( \pm 10.7) \\
\text { years }( \pm S D)\end{array}$ & CABG (elective) & $\begin{array}{l}\text { CK-MB, LDH } \\
\text { and CPK }\end{array}$ & $\begin{array}{l}3 / 2 \text { min, } 2 \text { cycles } \\
\text { (upper extremity) }\end{array}$ \\
\hline Wu, 2011 [27] & $25 / 25$ & $9 / 7$ & $\begin{array}{c}44.9( \pm 14.4) / 43.6 \\
( \pm 14.3), \text { years }( \pm S D)\end{array}$ & MVR (elective) & $\begin{array}{l}\text { Troponin I } \\
C_{\max } \text { within } \\
72 \text { hours }\end{array}$ & $\begin{array}{l}5 / 5 \text { min, } 3 \text { cycles } \\
\text { (upper extremity) and } \\
\text { 10/10 min, } 2 \text { cycles } \\
\text { (lower extremity) }\end{array}$ \\
\hline Hong, 2010 [20] & $65 / 65$ & $46 / 44$ & $\begin{array}{c}65.7( \pm 7.5) / 65.1( \pm 9.0) \\
\text { years }( \pm S D)\end{array}$ & OPCABG (elective) & $\begin{array}{l}\text { Troponin I } \\
\text { AUC after } \\
72 \text { hours }\end{array}$ & $\begin{array}{l}5 / 5 \text { min, } 4 \text { cycles } \\
\text { (upper extremity) }\end{array}$ \\
\hline Luo, 2011 [30] & $20 / 20$ & $13 / 8$ & $\begin{array}{c}2.8( \pm 1.0) / 2.7( \pm 0.9) \\
\text { years }( \pm S D)\end{array}$ & $\begin{array}{l}\text { VSD closure } \\
\text { (elective) }\end{array}$ & $\begin{array}{l}\text { Troponin I, } \\
\text { CK-MB }\end{array}$ & $\begin{array}{l}5 / 5 \text { min, } 3 \text { cycles } \\
\text { (lower extremity) }\end{array}$ \\
\hline $\begin{array}{l}\text { Karuppasamy, } \\
2011[23]\end{array}$ & $27 / 27$ & $22 / 23$ & $\begin{array}{c}66.9( \pm 11.2) / 67.3 \\
( \pm 10.3), \text { years }( \pm S D)\end{array}$ & $\begin{array}{l}\mathrm{CABG} \pm \mathrm{AVR} \\
\text { (elective) }\end{array}$ & $\begin{array}{l}\text { Troponin I } \\
\text { AUC after } \\
48 \text { hours }\end{array}$ & $\begin{array}{l}5 / 5 \text { min, } 3 \text { cycles } \\
\text { (upper extremity) }\end{array}$ \\
\hline
\end{tabular}

$C E A$ - carotid endarterectomy, $A U C$ - area under the curve, RIPC - remote ischemic preconditioning, AVR-aortic valve replacement, MVR - mitral valve replacement, $D V R$ - double valve replacement, ASD - atrial septal defect, VSD - ventricular septal defect, TGA - transposition of great vessels, ToF - tetralogy of Fallot,

$C K-M B$ - creatine kinase-myocardial band, CPK - creatine phosphokinase, $L D H$-lactate dehydrogenase

age of patients ranged from $160.83( \pm 58.39)$ days to 67 $( \pm 12)$ years in the rIPC group and from $154( \pm 56)$ days to $67( \pm 9)$ years among the controls. Male gender proportion ranged from $35 \%$ to $100 \%$ in the rIPC group and from $28 \%$ to $100 \%$ among the controls. The majority of patients were operated on due to coronary artery disease (335 in the rIPC group and 340 in the control group) [9, 20-27]. The analysis of rIPC in pediatric patients undergoing cardiac surgery included 67 patients in the rIPC study groups and 70 in the control groups [31-33], whereas 89 and 90 patients were submitted to isolated valvular surgery with or without rIPC, respectively [28-30]. All studies were proof-of-concept trials with small sample sizes, and only three included more than 100 patients [20-22]. Diabetic subjects were partially included in six trials [9, 21-24, 30]. Time from rIPC to global ischemia (e.g. cross-clamping of the aorta) was clearly stated in ten trials and equivocally in five. Two trials used the 'second window' of protection 
Table III. Characteristics of investigated trials

\begin{tabular}{|c|c|c|c|c|c|c|}
\hline Study/year/type & Exclusion/inclusion criteria & $\begin{array}{l}\text { Cross-clamp } \\
\text { time (min) }\end{array}$ & $\begin{array}{l}\text { Time from RIPC } \\
\text { to reperfusion }\end{array}$ & $\begin{array}{l}\text { Preoperative } \\
\text { LVEF (\%) } \\
\text { RIPC/Controls }\end{array}$ & $\begin{array}{l}\text { Myocardial } \\
\text { protection }\end{array}$ & $\begin{array}{l}\text { Jadad } \\
\text { Score - } \\
\text { (max. } 10 \\
\text { pts) }\end{array}$ \\
\hline $\begin{array}{l}\text { Cheung, } 2006 \\
\text { PRCT } \\
\text { Single-blinded [31] }\end{array}$ & $\begin{array}{l}\text { Excluded: isolated ASD, } \\
\text { Fontan completion, ToF, } \\
\text { chromosomal defect, airway } \\
\text { and parenchymal lung dise- } \\
\text { ase, immunodeficiency and } \\
\text { "blood disorders" }\end{array}$ & $55(13) / 59(13)$ & $\begin{array}{l}\text { Time from RIPC } \\
\text { to bypass: '5-10 } \\
\text { minutes'. }\end{array}$ & - & $\begin{array}{l}\text { Blood cardio- } \\
\text { plegia }\end{array}$ & 4 points \\
\hline $\begin{array}{l}\text { Zhou, } 2010 \\
\text { PRCT } \\
\text { Single-blinded [32] }\end{array}$ & $\begin{array}{l}\text { Included: patients with pul- } \\
\text { monary hypertension, VSD, } \\
\text { weight < } 7 \text { kg; Excluded: } \\
\text { heart failure, pneumonia, } \\
\text { history of other systemic } \\
\text { diseases, limb trauma, or } \\
\text { acidosis }\end{array}$ & $\begin{array}{c}24.13 \\
(9.83) / 24.17 \\
(8.21)\end{array}$ & $\begin{array}{l}\text { Repeated twice: } \\
24 \text { hours and } \\
1 \text { hour prior to } \\
\text { surgery }\end{array}$ & $\begin{array}{c}65.27 \\
(5.85) / 64.80 \\
(6.21)\end{array}$ & $\begin{array}{l}\text { Cardioplegic } \\
\text { arrest }\end{array}$ & 3 points \\
\hline $\begin{array}{l}\text { Wagner, } 2010 \\
\text { PRCT } \\
\text { Single-blinded [22] }\end{array}$ & $\begin{array}{l}\text { Excluded: age }>80 \text { years, } \\
\text { UA, LVEF }<30 \% \text {, CRF sCr } \\
>220 \mathrm{mmol} / \mathrm{l} \text {, bilirubin }>30 \\
\text { mmol/l, pulmonary disease, } \\
\text { recent systemic infection }\end{array}$ & $45 / 51$ & $\begin{array}{l}18(2) \text { hours prior } \\
\text { to surgery }\end{array}$ & $\begin{array}{l}<50 \%-20 / 21 \\
\text { patients }\end{array}$ & $\begin{array}{l}\text { Cold crystalloid } \\
\text { cardioplegia (St. } \\
\text { Thomas solution) }\end{array}$ & 7 points \\
\hline $\begin{array}{l}\text { Choi, } 2011 \\
\text { PRCT } \\
\text { Single-blinded [29] }\end{array}$ & $\begin{array}{l}\text { Excluded: age }>80 \text { years, } \\
\text { LM (LCA) stenosis }>50 \% \text {, } \\
\text { hepatic or pulmonary } \\
\text { disease, active infective } \\
\text { endocarditis, LVEF }<30 \% \text {, } \\
\text { AMI within } 3 \text { weeks, sCr } \\
>1.4 \text { (female) or } 1.6 \text { (male) } \\
\text { mg/dl, PDA }\end{array}$ & $\begin{array}{c}98(27) / 108 \\
(29)\end{array}$ & $\begin{array}{l}\text { At least } 10 \mathrm{~min}- \\
\text { utes }\end{array}$ & $63(9) / 60(12)$ & $\begin{array}{l}\text { Blood cardio- } \\
\text { plegia }\end{array}$ & 6 points \\
\hline $\begin{array}{l}\text { Li, } 2010 \\
\text { PRCT } \\
\text { Single-blinded [28] }\end{array}$ & $\begin{array}{l}\text { Excluded: age > } 65 \text { years, } \\
\text { non-rheumatic heart valve } \\
\text { disease, infective endocardi- } \\
\text { tis, previous cardiac surgery, } \\
\text { diabetes mellitus, CAD, PDA, } \\
\text { arterial hypertension, ASA, } \\
\text { ACE inhibitors or corticoste- } \\
\text { roids or statins }\end{array}$ & $\begin{array}{c}47.3 \\
(17.9) / 47.4 \\
(17.3)\end{array}$ & $\begin{array}{l}\text { After the induc- } \\
\text { tion of anesthesia }\end{array}$ & $<55 \%-7 / 6$ & $\begin{array}{l}\text { Cold blood car- } \\
\text { dioplegia }\end{array}$ & 5 points \\
\hline $\begin{array}{l}\text { Hausenloy, } 2007 \\
\text { PRCT } \\
\text { Single-blinded [9] }\end{array}$ & $\begin{array}{l}\text { Excluded: age }>80 \text { years, } \\
\text { UA, LM (LCA) stenosis, } \\
\text { hepatic, renal, pulmonary } \\
\text { disease, PDA of upper limbs } \\
\text { or on oral hypoglycemics }\end{array}$ & $\begin{array}{c}36(17) / 45 \\
(22)\end{array}$ & $\begin{array}{l}\text { Not more than } 45 \\
\text { minutes before } \\
\text { aortic cross- } \\
\text { clamping }\end{array}$ & $<55 \%-5 / 10$ & $\begin{array}{l}\text { Cardioplegic } \\
\text { arrest and } \\
\text { intermittent } \\
\text { cross-clamp } \\
\text { fibrillation }\end{array}$ & 7 points \\
\hline $\begin{array}{l}\text { Rahman, } 2010 \\
\text { PRCT } \\
\text { Double-blinded [19] }\end{array}$ & $\begin{array}{l}\text { Included: elective and } \\
\text { urgent patients; Excluded: } \\
\text { UA within } 48 \text { hours, AMI } \\
\text { within } 30 \text { days, pregnancy, } \\
\text { DM, dialysis, other than } \\
\text { CABG, radial artery usage }\end{array}$ & $76(21) / 71(18)$ & $74(16) \min$ & $\begin{array}{c}58.5(13.3) / 61.6 \\
(13.4)\end{array}$ & $\begin{array}{l}\text { Intermittent cold } \\
\text { blood cardio- } \\
\text { plegia }\end{array}$ & $\begin{array}{c}9 \\
\text { points }\end{array}$ \\
\hline $\begin{array}{l}\text { Ali, } 2010 \\
\text { PRCT } \\
\text { Single-blinded [21] }\end{array}$ & $\begin{array}{l}\text { Excluded: significant renal } \\
\text { and hepatic disease, hemo- } \\
\text { dynamic instability, UA, AMI } \\
\text { within } 4 \text { weeks or ongoing }\end{array}$ & $\begin{array}{c}33.4 \\
(10.6) / 33.7 \\
(8.5)\end{array}$ & $\begin{array}{l}\text { Before placing } \\
\text { the patient on } \\
\text { bypass }\end{array}$ & $\begin{array}{l}<55 \%- \\
46 \% / 27 \%\end{array}$ & $\begin{array}{l}\text { Warm blood } \\
\text { cardioplegia }\end{array}$ & $\begin{array}{c}5 \\
\text { points }\end{array}$ \\
\hline $\begin{array}{l}\text { Thielmann, } 2010 \\
\text { PRCT } \\
\text { Single-blinded [25] }\end{array}$ & $\begin{array}{l}\text { Excluded: DM, renal failure, } \\
\text { PDA of upper extremities, } \\
\text { hemodynamic instability, } \\
\text { AMI within the previous } \\
2 \text { weeks, emergency, com- } \\
\text { bined or redo surgery }\end{array}$ & $54(14) / 53(11)$ & $54(14) \min$ & $\begin{array}{l}<55 \%- \\
19 \% / 23 \%\end{array}$ & $\begin{array}{l}\text { Cold crystalloid } \\
\text { cardioplegia }\end{array}$ & 6 points \\
\hline $\begin{array}{l}\text { Venugopal, } 2009 \\
\text { PRCT } \\
\text { Single-blinded [24] }\end{array}$ & $\begin{array}{l}\text { Excluded: age }>80 \text { years, } \\
\text { UA, DM, hepatic, renal or } \\
\text { pulmonary disease, PDA of } \\
\text { upper limbs }\end{array}$ & $\begin{array}{c}53(14) / 65 \\
(30)\end{array}$ & $\begin{array}{l}60 \text { minutes before } \\
\text { aortic cross- } \\
\text { clamping }\end{array}$ & $<55 \%-3 \% / 1 \%$ & $\begin{array}{l}\text { Cold blood car- } \\
\text { dioplegia }\end{array}$ & $\begin{array}{c}7 \\
\text { points }\end{array}$ \\
\hline
\end{tabular}


Table III. Cont.

\begin{tabular}{|c|c|c|c|c|c|c|}
\hline Study/year/type & Exclusion/inclusion criteria & $\begin{array}{l}\text { Cross-clamp } \\
\text { time (min) }\end{array}$ & $\begin{array}{l}\text { Time from RIPC } \\
\text { to reperfusion }\end{array}$ & $\begin{array}{l}\text { Preoperative } \\
\text { LVEF (\%) } \\
\text { RIPC/Controls }\end{array}$ & $\begin{array}{l}\text { Myocardial } \\
\text { protection }\end{array}$ & $\begin{array}{l}\text { Jadad } \\
\text { Score - } \\
\text { (max. } 10 \\
\text { pts) }\end{array}$ \\
\hline $\begin{array}{l}\text { Gunaydin, } 2000 \\
\text { PRCT } \\
\text { Single-blinded [26] }\end{array}$ & $\begin{array}{l}\text { Excluded: UA, DM, LV aneu- } \\
\text { rysm, LVEF < } 40 \%\end{array}$ & $\begin{array}{c}37.8 \\
(27.0) / 28.5 \\
(11.4)\end{array}$ & $\begin{array}{l}2 \text { minutes before } \\
\text { aortic cross- } \\
\text { clamping }\end{array}$ & $\begin{array}{c}\text { Not lower than } \\
40 \%\end{array}$ & $\begin{array}{l}\text { Blood cardio- } \\
\text { plegia }\end{array}$ & 3 points \\
\hline $\begin{array}{l}\text { Wu, } 2011 \\
\text { PRCT } \\
\text { Single-blinded [27] }\end{array}$ & $\begin{array}{l}\text { Excluded: concomitant } \\
\text { heart abnormalities, } \\
\text { NYHA 4, history of respira- } \\
\text { tory infection, asthma or } \\
\text { previous cardiac surgery, } \\
\text { hepatic, renal, pulmonary } \\
\text { disease. PDA and usage of } \\
\text { oral hypoglycemics }\end{array}$ & $\begin{array}{c}63.5 \\
(19.8) / 51.7 \\
(7.4)\end{array}$ & $\begin{array}{l}\text { After the induc- } \\
\text { tion of anesthesia }\end{array}$ & $\begin{array}{c}61.1(8.0) / 63.4 \\
(7.0)\end{array}$ & $\begin{array}{l}\text { Cold blood car- } \\
\text { dioplegia }\end{array}$ & 5 points \\
\hline $\begin{array}{l}\text { Hong, } 2010 \\
\text { PRCT } \\
\text { Double-blinded [20] }\end{array}$ & $\begin{array}{l}\text { Excluded: age > } 80 \text { years, } \\
\text { UA, heart failure requiring } \\
\text { mechanical or inotropic } \\
\text { support, combined surgery, } \\
\text { sever renal, liver or pulmo- } \\
\text { nary disease, LVEF < 30\%, } \\
\text { AMI or sepsis/infection } \\
\text { within } 7 \text { days, Nicorandil us- } \\
\text { age, PDA, or amputation }\end{array}$ & - & $19.2(10.8) \mathrm{min}$ & $\begin{array}{c}56.6(10) / 53.5 \\
(12.2)\end{array}$ & $\begin{array}{l}\text { Intra-coronary } \\
\text { shunt }\end{array}$ & 10 points \\
\hline $\begin{array}{l}\text { Luo, } 2011 \\
\text { PRCT } \\
\text { Single-blinded [30] }\end{array}$ & $\begin{array}{l}\text { Included: elective surge- } \\
\text { ry, aged } 1 \text { to } 5 \text { years old; } \\
\text { Excluded: concomitant } \\
\text { surgery, infective endocar- } \\
\text { ditis, preoperative medica- } \\
\text { tions usage, hepatic or renal } \\
\text { malfunctioning }\end{array}$ & $\begin{array}{c}36.1 \\
(15.8) / 32.7 \\
(12.4)\end{array}$ & $\begin{array}{l}\text { Immediately after } \\
\text { the induction of } \\
\text { anesthesia }\end{array}$ & - & $\begin{array}{l}\text { Cold blood car- } \\
\text { dioplegia }\end{array}$ & 4 points \\
\hline $\begin{array}{l}\text { Karuppasamy, } 2011 \\
\text { Single-blinded [23] }\end{array}$ & $\begin{array}{l}\text { Excluded: age > } 85 \text { years, } \\
\text { UA, hepatic, pulmonary, } \\
\text { renal disease, PDA affecting } \\
\text { upper limbs, receiving oral } \\
\text { hypoglycemics }\end{array}$ & $\begin{array}{c}43.4 \\
(15.2) / 56.6 \\
(27.1)\end{array}$ & $\begin{array}{l}\text { After the induc- } \\
\text { tion of anesthesia }\end{array}$ & $\begin{array}{c}54.0(12.1) / 54.4 \\
(1.5)\end{array}$ & $\begin{array}{l}\text { Intermittent } \\
\text { aortic cross- } \\
\text { clamping and } \\
\text { intermittent cold } \\
\text { blood cardio- } \\
\text { plegia }\end{array}$ & 7 points \\
\hline
\end{tabular}

(rIPC performed $>24$ hours before the ischemic insult), whereas the rest used the 'immediate window' of protection (around 60 minutes from the insult).

\section{Meta-analysis of RCTs according to outcomes of interest}

\section{Postoperative release of myocardial injury markers}

Our analysis found that rIPC was associated with the reduction of myocardial injury markers by -0.63 SMD $(-0.63$, $95 \% \mathrm{Cl}:-0.99$ to $-0.28 ; p<0.0005)$ in the adult patients and by -1.19 SMD $(-1.19,95 \% \mathrm{Cl}:-1.56$ to $-0.82 ; p<0.00001)$ in the pediatric population. The degree of heterogeneity was exceptionally high $\left(1^{2}=83 \%\right)$ for the adult group and extremely low for the pediatric population $\left(\mathrm{I}^{2}=0 \%\right)$. The analysis included 424 adult and 67 pediatric patients in the rIPC group. This model was assessed for the whole population of adult cardiac surgery patients, including coronary and valvular disease. Separate analysis was performed for pediatric cardiac procedures (Fig. 2). The analysis of bias was performed by means of funnel plot asymmetry assessment, as presented in Fig. 3.

\section{Perioperative inotropic support requirement}

Adult patients receiving IIPC benefited from lower inotropic support requirement following cardiac surgery, as shown by the negative SMD $(-0.40,95 \% \mathrm{Cl}:-0.66$ to $-0.14 ; p<0.002)$. This effect was even more pronounced in children undergoing heart surgery and $\mathrm{rIPC}(-0.83,95 \% \mathrm{Cl}$ : -1.18 to $-0.48 ; p<0.00001)$. This analysis included 116 adult and 67 pediatric patients in the rIPC group with the exceptionally low heterogeneity of $0 \%$ (Fig. 4).

\section{Postoperative ICU stay}

No benefit was found for either adult or pediatric patients receiving rIPC with regard to ICU length of stay. This 


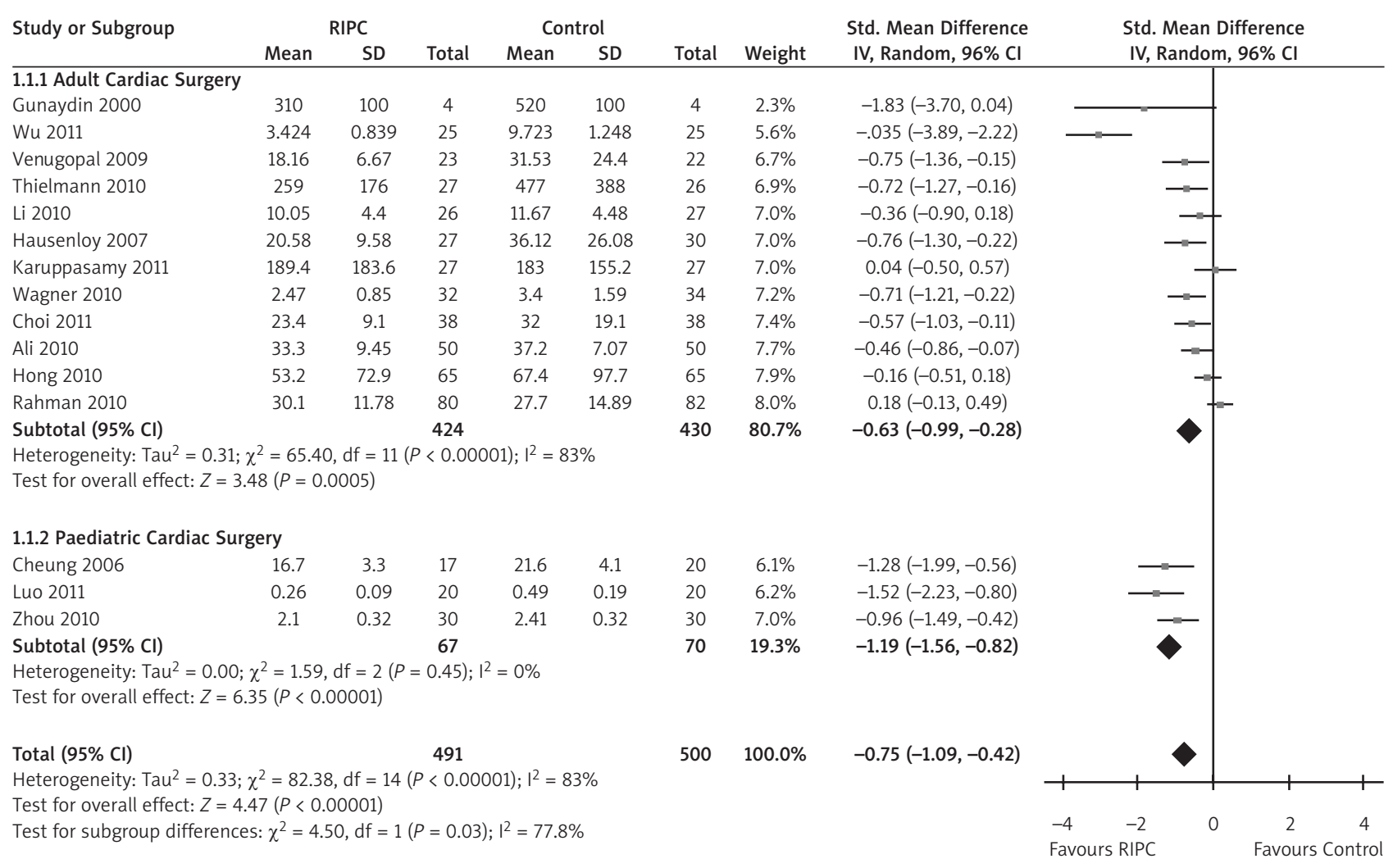

Fig. 2. Myocardial injury markers

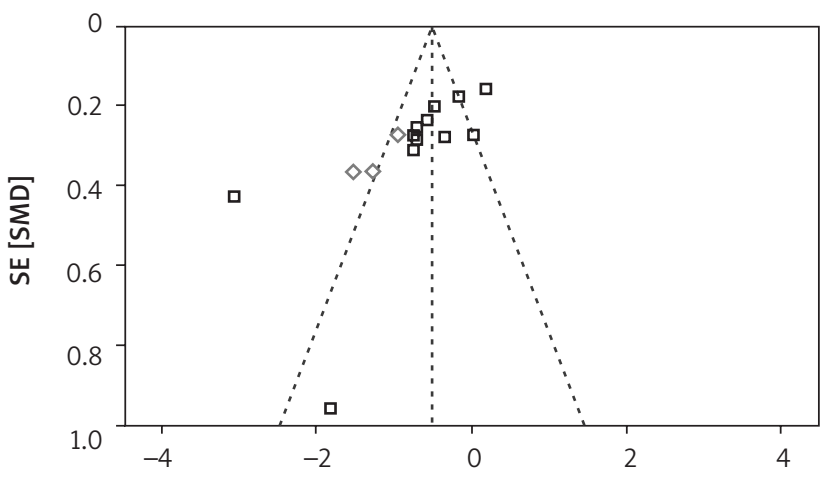

Adult Cardiac Surgery $\diamond$ Paediatric Cardiac Surgery

Fig. 3. Funnel plot - studies included in myocardial injury markers analysis

end-point was burdened with moderate heterogeneity $\left(1^{2}=40 \%\right)$. The MD in the adults $(-0.63$ hour $95 \% \mathrm{Cl}:-4.60$ to $3.34 ; p=0.76)$ and in the pediatric group $(-0.59$ hour $95 \% \mathrm{Cl}:-7.29$ to $6.10 ; p=0.86$ ) did not favor rIPC (Fig. 5).

\section{Bias and heterogeneity assessment Degree of bias}

All studies which were found eligible for the review were in fact of prospective randomized design, which is known to provide the most trustworthy evidence. Among these studies, we found discrepancies in the quality of reporting and methodological design. Firstly, all studies but two were single-blind, as it was extremely difficult to provide satis- factory logistics for a double-blind study with rIPC. Only Rahman et al. and Hong et al. managed to provide such an environment for their true double-blind studies. Secondly, this meta-analysis gathered its evidence from proofof-concept trials, which are, by definition, limited in terms of the number of participants. Funnel plot analysis was employed to find evidence of asymmetry, and four studies were indeed found to lie outside the $95 \%$ confidence interval, with one additional study located on its border [20, 21, 24, 28, 31] (Fig. 3). The risk of bias is presented as percentages across all studies in Fig. 6, whereas individual domain-based assessment is provided in Fig. 7. Jadad score calculation stratified the studies into two groups with high and low risk of bias, with a clear cut-off point of 5 points and above for low risk studies. Table I provides data on individual Jadad scoring.

\section{Sensitivity analysis}

The application of a fixed effect model did not change the results significantly, but the effect of rIPC on the lowering of postoperative myocardial necrosis markers appeared smaller in the adult group (SMD $-0.40 ; 95 \% \mathrm{Cl}$ : -0.54 to $-0.27 ; p=0.00001$ ). Subgroup analysis of the studies reporting outcomes of CABG with or without AVR showed results consistent with the general trend (SMD $-0.42 ; 95 \% \mathrm{Cl}:-0.70$ to $-0.13, p=0.005)$ and $\mathrm{I}^{2}=67 \%[9,20-27]$. Studies concerning isolated valvular surgery did not confirm the protective result of rIPC; however, heterogeneity $\left(1^{2}=94 \%\right)$ was essentially influencing the outcome effect under the random 


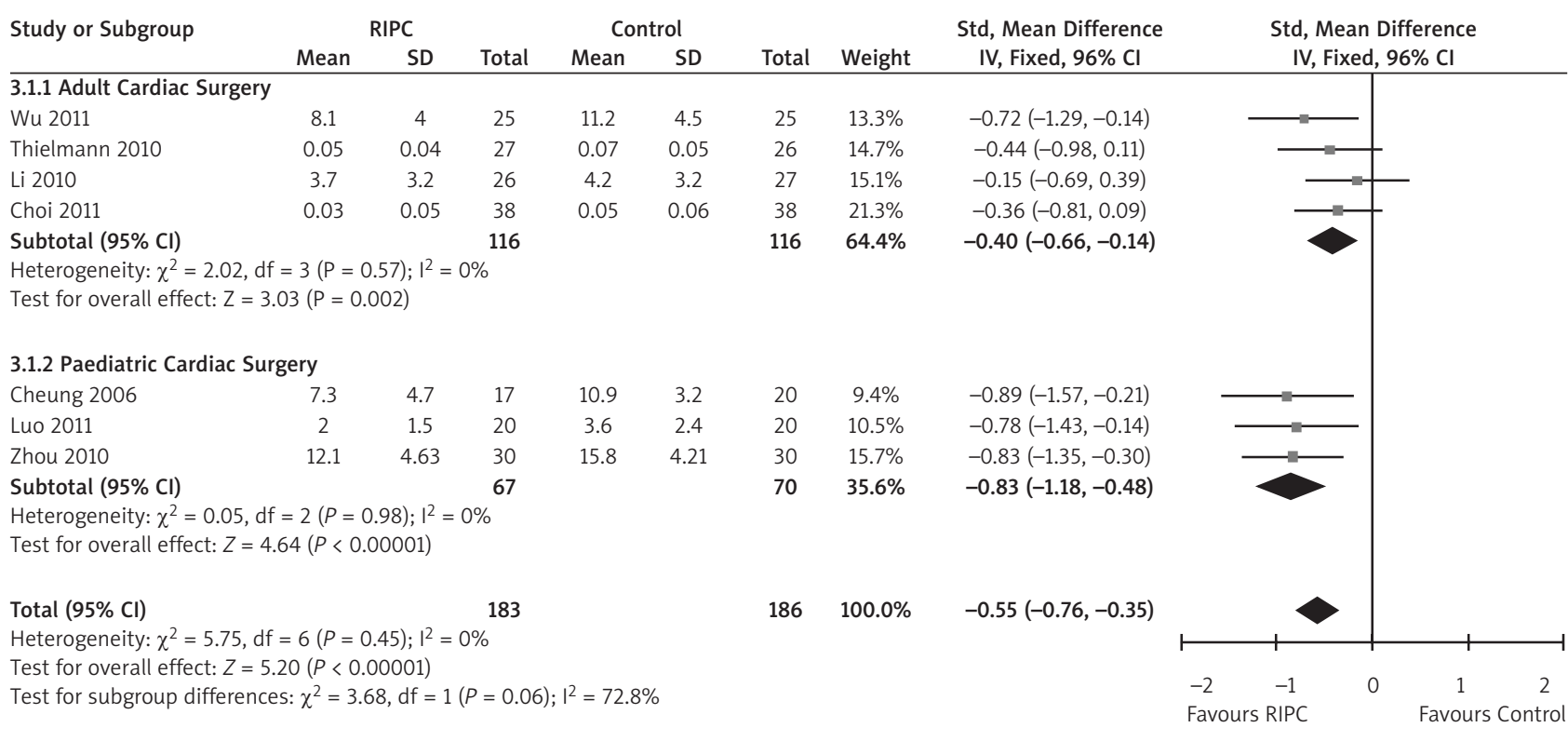

Fig. 4. Inotropic support requirements

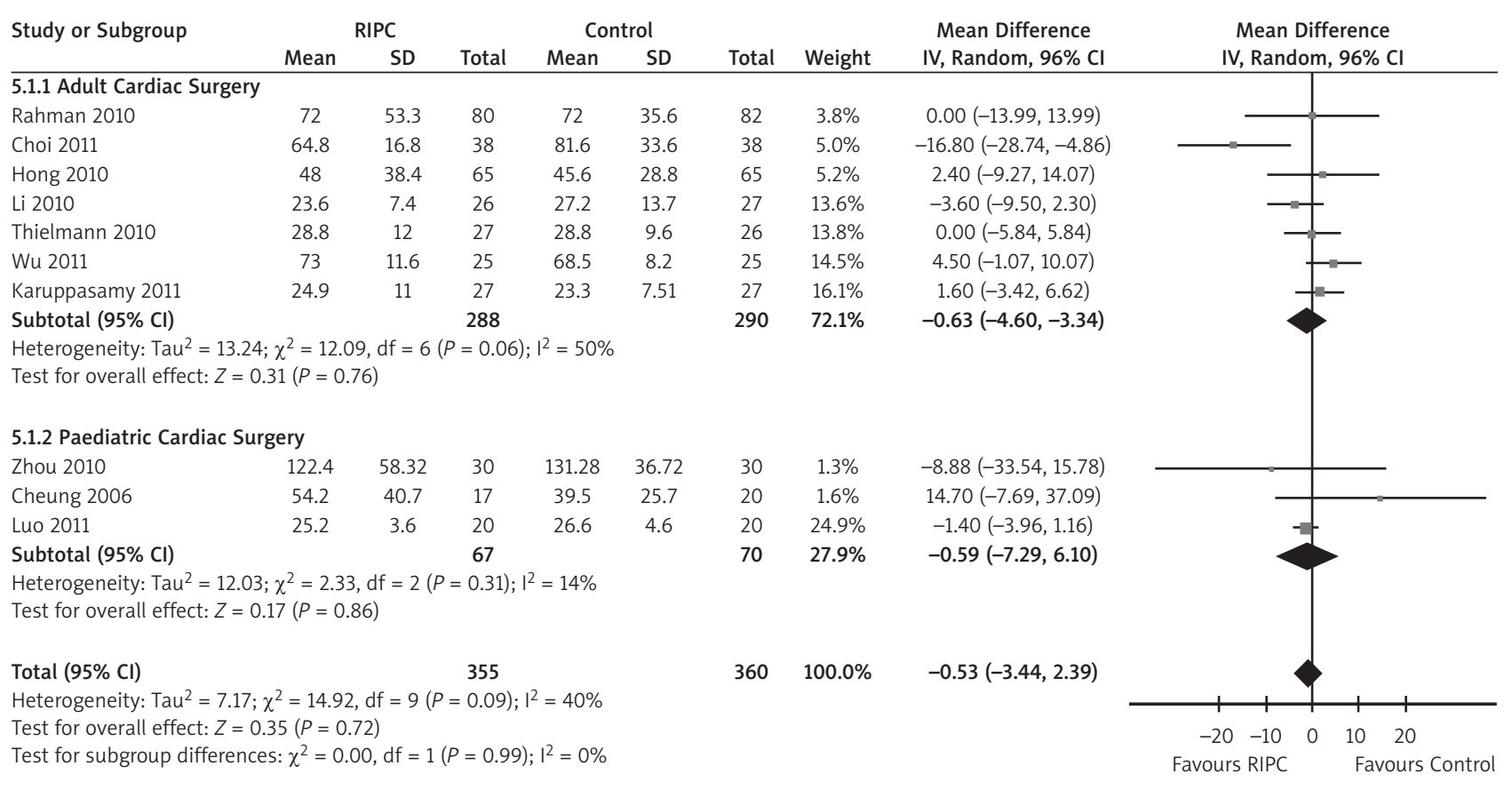

Fig. 5. Length of ICU stay in the postoperative period

model (SMD -1.28; $95 \% \mathrm{Cl}:-2.63$ to $0.07 ; p=0.06$ ), and it changed when a fixed model was used (SMD $-0.87 ; 95 \% \mathrm{Cl}$ : -1.19 to $-0.54 ; p<0.00001$ ) [28-30]. Relevant outcomes associated with rIPC were found in patients undergoing pediatric cardiac surgery without heterogeneity $\left(\mathrm{I}^{2}=0 \%\right)(\mathrm{SMD}$ $-1.19 ; 95 \% \mathrm{Cl}:-1.56$ to $-0.82 ; p=0.00001$ ) [30-32]. When the rIPC protocol was performed in the anatomic region of the lower extremities, the result was significant (SMD -1.31 ; 95\% Cl: 2.14 to $0.48 ; p=0.002$ ) with peaked heterogeneity $\left(\mathrm{I}^{2}=88 \%\right)$. When $\mathrm{rIPC}$ was applied to an upper extremity, the effect appeared smaller, but still significant (SMD -0.54 ; $95 \% \mathrm{Cl}:-0.84$ to $-0.23 ; p=0.0006)$, and $\mathrm{I}^{2}$ was $71 \%$.
In order to correct for the high heterogeneity in the whole population, we restricted the analysis to studies lying inside the $95 \% \mathrm{Cl}$ on the funnel plot, excluding five studies [20, 21, $24,28,31]$. This correction changed the heterogeneity from $83 \%$ to $0 \%$ and the effect was still highly significant (SMD $-0.69 ; 95 \% \mathrm{Cl}:-0.86$ to $-0.51 ; p=0.00001)$. With regard to studies scoring $>5$ points on the Jadad scale, the meta-analysis showed a significant reduction of necrosis markers, although the effect was smaller than the effect for the whole population (SMD $-0.40 ; 95 \% \mathrm{Cl}:-0.69$ to $-0.11 ; p=0.005$ ) [9, $20,21,23-26,30]$. We analyzed the effect of rIPC on diabetic patients separately, and compared it with trials in which 


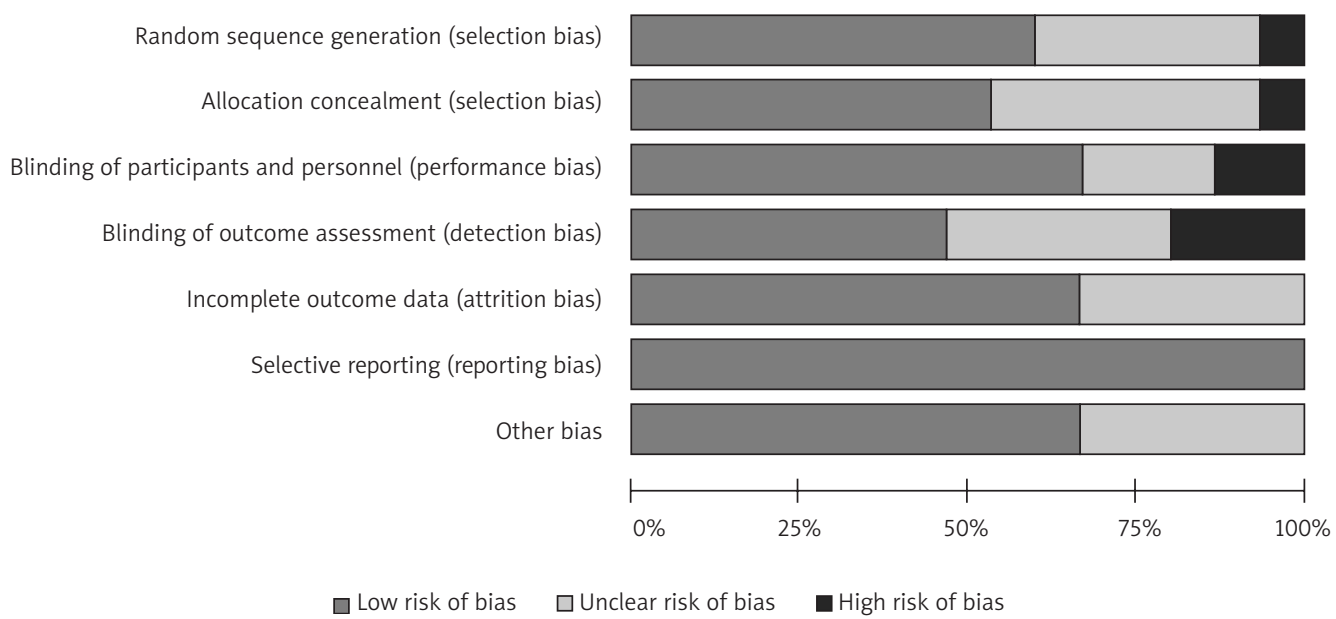

Fig. 6. Risk of bias assessment. Six domains used for the individual judging

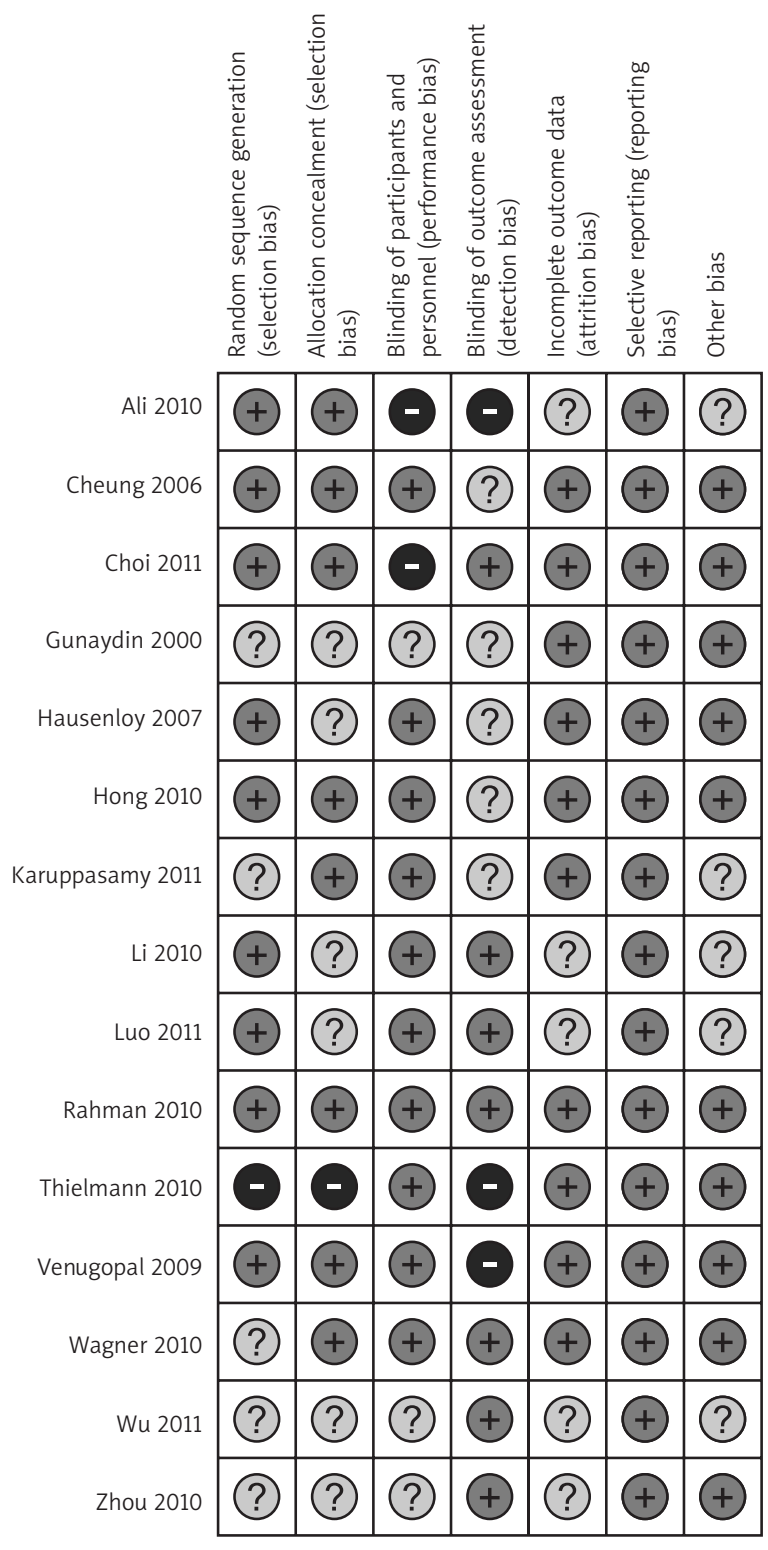

Fig. 7. Risk of bias assessment. Individual domain-based analysis non-diabetic patients were enrolled. For diabetic patients there was a significant, though modest, reduction of myocardial necrosis markers, whereas for non-diabetic patients this effect was large and significant (diabetic: SMD -0.42 ; 95\% Cl: -0.66 to $-0.19 ; p=0.0005$ and $\mathrm{I}^{2}=40 \%$; non-diabetic: SMD $-1.06 ; 95 \% \mathrm{Cl}:-1.66$ to $-0.45 ; p=0.0006$ and $\left.\mathrm{I}^{2}=89 \%\right)[9,21-24,30]$.

\section{Discussion}

The results of the analysis appear to suggest that remote ischemic preconditioning is indeed associated with the reduction of the postoperative biomarkers of myocardial injury (Fig. 2). The included evidence is recent and welldesigned, yet the number of participants in all the studies and their proof-of-concept character might limit their usability as final evidence. Our analysis suggests several clear-cut points. The impact of rIPC on ischemia-reperfusion injury is detectable clinically, and in some studies its magnitude is very high [9]. Not only is direct myocardial necrosis limited, but so is myocardial stunning, as can be assessed by the lower requirement of inotropic support in patients submitted to the intervention. Sensitivity analysis provided new insights which cannot be gained in the big picture. The more pronounced effect of rIPC in pediatric cardiac surgery might be associated with the lack of coronary disease, young age, or specific features of congenital lesions. We could not venture any conclusions concerning the insignificant effect of rIPC in valve surgery, as the heterogeneity was abnormally high and could not be corrected. After a recent study by Wu et al., we hypothesized that technical nuances, such as the anatomic region in which the rIPC protocol is being executed, might play a role in shaping the magnitude of the final effect [28]. To our surprise, when applied in the leg, rIPC was associated with an almost three times greater effect in terms of reducing myocardial injury. Last, but not least, we noted a severe reduction in the cardioprotective benefit of rIPC in the population of patients suffering from diabetes mellitus. 
According to our review, four studies included in the analysis reported no benefit from $\mathrm{rIPC}$ in the setting of cardiac surgery [20, 21, 24, 29]. In all studies but one, the upper extremity was used for the provision of the remote ischemic stimulus. Two of the four studies used pulse oximeters to ascertain that no collateral flow was present during the execution of the protocol. Hong et al. and Karuppasamy et al. enrolled diabetic patients. Additionally, Rahaman et al. executed the rIPC protocol with the longest time period from rIPC to ischemic insult, $74( \pm 16)$ minutes, while Hong et al. managed to provide the whole protocol in just $19( \pm 11)$ minutes prior to the insult; neither of them managed to find rIPC of cardioprotective value. Hong et al. hypothesized that in off-pump CABG, with intracoronary shunt deployment, there is too little damage to the myocardium due to ischemia and reperfusion; therefore, even if statistically insignificant, the reduction of $26 \%$ of cTnI AUC over 72 hours was indeed clinically relevant in this individual case. It still remains unknown whether these factors played any role in the negative results in the aforementioned studies.

The effect of rIPC was found to be the most pronounced among pediatric patients operated on due to congenital cardiac anomalies (mainly VSDs). Nevertheless, the effect seemed proportionally small. This can be explained by the generally low-risk population included in proof-of-concept trials. Only Rahman et al. enrolled emergency CABG patients, who are, by definition, not the most suitable subjects for studying the effect of any intervention. As observed by Peters, the studies comparing the effects of rIPC in fact include very complex populations, bearing a myriad of confounding morbidities [34]. Therefore, we would like to propose a standardization of subjects that might bear more resemblance to the 'real-life' population and in whom the benefit of rIPC would be the most visible. We postulate that the most suitable population for future research would be a high-risk population of patients submitted to non-emergency on-pump CABG with the use of intermittent cold blood cardioplegia, aged fifty years and older, especially if the patients are burdened with one or more of the following risk factors: female gender, history of previous myocardial infarction (more than seven days but less than two years before surgery), LVEF lower than 30\%, previous ischemic stroke, transient ischemic attack, or diabetes mellitus. In such a population, relevant end-points of all-cause mortality, perioperative cerebrovascular accident (CVA), PMI, or the need for mechanical support would be easy to assess, and the question whether rIPC is genuinely efficacious in heart protection could be answered. Additionally, we do not recommend using volatile anesthetics, as these might reduce the overall benefit of rIPC by mechanisms that are not entirely recognized [34]. We encourage the use of the lower extremities for the provision of the rIPC stimulus, as this has shown a greater magnitude of protective effect in several of the aforementioned studies [21].

\section{Comparison of our study with other meta-analyses}

Takagi et al. authored the first meta-analysis rendering comparable results, yet he only assessed myocardial necrosis markers and combined outcomes from cardiac and vascular surgery, with a modest number of patients submitted to $\operatorname{rIPC}[12,13,35]$. We believe that the sensitivity analysis and nearly one thousand patients included in our analysis, along with an additional risk of bias assessment, makes this document the primary source of evidence for the usefulness of rIPC in cardiac surgery.

\section{Study strengths and limitations}

There is a tendency among small studies to render a large treatment effect associated with the studied intervention; we could not overcome this problem, because large clinical studies have not come into existence so far. Moreover, we understand the limitation of meta-analytical approaches in combining only published data, which, inevitably, introduces bias in contemporary reviews. High heterogeneity and the mixed population enrolled were overcome by using a random effect model, subgroup analysis, and scrutiny of symmetry in the analysis of the funnel plot. We could not provide more clinically relevant outcomes, such as perioperative myocardial infarction, in-hospital mortality, and long-term mortality, as these are still unavailable. Instead, we used surrogate outcomes, such as myocardial necrosis markers release in lieu of perioperative myocardial necrosis and inotropic support requirement in lieu of low-output syndrome due to myocardial stunning, as these outcomes are associated with long-term prognosis for patients submitted to cardiac surgery [36].

\section{Future research}

Multicenter studies enrolling 'real-life' populations in a prospective, randomized manner are currently taking place. These studies will assess clinically relevant endpoints such as PMIs and in-hospital mortality. The longterm effects of rIPC in cardiac surgery should be studied thereafter.

\section{Conclusions}

This meta-analysis provides evidence that rIPC should be considered as a tool for providing myocardial protection in cardiac surgery patients, with all limitations characteristic of proof-of-concept trials, upon which the evidence was built.

\section{Acknowledgements}

We thank the following authors for their cooperation during the creation of this review: Dr Robert Wagner (Brno, Czech Republic), Dr Hisato Takagi (Nagasawa, Japan), Dr Shanglong Yao (Jiefang Da Dao, China), Dr Wanjun Luo (Changsha, China), and Dr Jeon Yun Seok (Seoul, Korea). 


\section{References}

1. Maroko PR, Libby P, Ginks WR, Bloor CM, Shell WE, Sobel BE, Ross J Jr. Coronary artery reperfusion. I. Early effects on local myocardial function and the extent of myocardial necrosis. J Clin Invest 1972; 51: 2710-2716.

2. Garcia-Dorado D, Ruiz-Meana M, Piper HM. Lethal reperfusion injury in acute myocardial infarction: facts and unresolved issues. Cardiovasc Res 2009; 83: 165-168.

3. Venugopal V, Ludman A, Yellon DM, Hausenloy DJ. 'Conditioning' the heart during surgery. Eur J Cardiothorac Surg 2009; 35: 977-987.

4. Kharbanda RK, Mortensen UM, White PA, Kristiansen SB, Schmidt MR, Hoschtitzky JA, Vogel M, Sorensen K, Redington AN, MacAllister R. Transient limb ischemia induces remote ischemic preconditioning in vivo. Circulation 2002; 106: 2881-2883.

5. Konstantinov IE, Arab S, Kharbanda RK, Li J, Cheung MM, Cherepanov V, Downey GP, Liu PP, Cukerman E, Coles JG, Redington AN. The remote ischemic preconditioning stimulus modifies inflammatory gene expression in humans. Physiol Genomics 2004; 19: 143-150.

6. Konstantinov IE, Li J, Cheung MM, Shimizu M, Stokoe J, Kharbanda RK, Redington AN. Remote ischemic preconditioning of the recipient reduces myocardial ischemia-reperfusion injury of the denervated donor heart via a Katp channel-dependent mechanism. Transplantation 2005; 79 . 1691-1695.

7. Marczak J, Nowicki R, Kulbacka J, Saczko J. Is remote ischaemic preconditioning of benefit to patients undergoing cardiac surgery? Interact Cardiovasc Thorac Surg 2012; 14: 634-639.

8. Ludman AJ, Yellon DM, Hausenloy DJ. Cardiac preconditioning for ischaemia: lost in translation. Dis Model Mech 2010; 3: 35-38.

9. Hausenloy DJ, Mwamure PK, Venugopal V, Harris J, Barnard M, Grundy E, Ashley E, Vichare S, Di Salvo C, Kolvekar S, Hayward M, Keogh B, MacAllister RJ, Yellon DM. Effect of remote ischaemic preconditioning on myocardial injury in patients undergoing coronary artery bypass graft surgery: a randomised controlled trial. Lancet 2007; 370: 575-579.

10. Kharbanda RK, Nielsen TT, Redington AN. Translation of remote ischaemic preconditioning into clinical practice. Lancet 2009; 374: 1557-1565.

11. Takagi H, Umemoto T. Remote ischemic preconditioning for cardiovascular surgery: an updated meta-analysis of randomized trials. Vasc Endovascular Surg 2011; 45: 511-513.

12. Takagi H, Manabe H, Kawai N, Goto S, Umemoto T. Review and meta-analysis of randomized controlled clinical trials of remote ischemic preconditioning in cardiovascular surgery. Am J Cardiol 2008; 102: 1487-1488.

13. Ludman AJ, Hausenloy DJ, Venugopal V, Yellon DM. Premature meta-analysis of remote ischemic preconditioning. Am J Cardiol 2009; 103: 893-894.

14. Jadad AR, Moore RA, Carroll D, Jenkinson C, Reynolds DJ, Gavaghan DJ, MCQuay HJ. Assessing the quality of reports of randomized clinical trials: is blinding necessary? Control Clin Trials 1996; 17: 1-12.

15. Wiebe N, Vandermeer B, Platt RW, Klassen TP, Moher D, Barrowman NJ. A systematic review identifies a lack of standardization in methods for handling missing variance data. J Clin Epidemiol 2006; 59: 342-353.

16. Moher D, Liberati A, Tetzlaff J, Altman DG, Group P. Preferred reporting items for systematic reviews and meta-analyses: the PRISMA statement. Int J Surg 2010; 8: 336-341.

17. Higgins J, Green S. Cochrane for systematic reviews of interventions. John Wiley \& Sons Ltd., Chichester 2009.

18. Review Manager (RevMan) [Computer program]. Version 5.1. Copenhagen: The Nordic Cochrane Centre, The Cochrane Collaboration, 2011.

19. Higgins JP, Thompson SG, Deeks JJ, Altman DG. Measuring inconsistency in meta-analyses. BMJ 2003; 327: 557-560.

20. Rahman IA, Mascaro JG, Steeds RP, Frenneaux MP, Nightingale P, Gosling P, Townsend P, Townend JN, Green D, Bonser RS. Remote ischemic preconditioning in human coronary artery bypass surgery from promise to disappointment? Circulation 2010; 122: S53-S59.
21. Hong DM, Min JJ, Kim JH, Sohn IS, Lim TW, Lim YJ, Bahk JH, Jeon Y. The effect of remote ischaemic preconditioning on myocardial injury in patients undergoing off-pump coronary artery bypass graft surgery. Anaesth Intensive Care 2010; 38: 924-929.

22. Ali N, Rizwi F, Iqbal A, Rashid A. Induced remote ischemic pre-conditioning on ischemia-reperfusion injury in patients undergoing coronary artery bypass. J Coll Physicians Surg Pak 2010; 20: 427-431.

23. Wagner R, Piler P, Bedanova H, Adamek P, Grodecka L, Freiberger T. Myocardial injury is decreased by late remote ischaemic preconditioning and aggravated by tramadol in patients undergoing cardiac surgery: a randomised controlled trial. Interact Cardiovasc Thorac Surg 2010; 11: 758-762.

24. Karuppasamy P, Chaubey S, Dew T, Musto R, Sherwood R, Desai J, John L, Shah AM, Marber MS, Kunst G. Remote intermittent ischemia before coronary artery bypass graft surgery: a strategy to reduce injury and inflammation? Basic Res Cardiol 2011; 106: 511-519.

25. Venugopal V, Hausenloy DJ, Ludman A, Di Salvo C, Kolvekar S, Yap J, Lawrence $D$, Bognolo J, Yellon DM. Remote ischaemic preconditioning reduces myocardial injury in patients undergoing cardiac surgery with cold-blood cardioplegia: a randomised controlled trial. Heart (London) 2009; 95: 1567-1571.

26. Thielmann M, Kottenberg E, Boengler K, Raffelsieper C, Neuhaeuser M, Peters J, Jakob $\mathrm{H}$, Heusch $\mathrm{G}$. Remote ischemic preconditioning reduces myocardial injury after coronary artery bypass surgery with crystalloid cardioplegic arrest. Basic Res Cardiol 2010; 105: 657-664.

27. Günaydin B, Cakici I, Soncul H, Kalaycioglu S, Cevik C, Sancak B, Kanzik I, Karadenizli Y. Does remote organ ischaemia trigger cardiac preconditioning during coronary artery surgery? Pharmacol Res 2000; 41: 493-496.

28. Wu Q, Gui P, Wu J, Ding D, Purusram G, Dong N, Yao S. Effect of limb ischemic preconditioning on myocardial injury in patients undergoing mitral valve replacement surgery. Circ J 2011; 75: 1885-1889.

29. Li L, Luo WJ, Huang LJ, Zhang WX, Gao Y, Jiang HH, Zhang C, Long L, Chen S. Remote perconditioning reduces myocardial injury in adult valve replacement: a randomized controlled trial. J Surg Res 2010; 164: E21-E26.

30. Choi YS, Shim JK, Kim JC, Kang KS, Seo YH, Ahn KR, Kwak YL. Effect of remote ischemic preconditioning on renal dysfunction after complex valvular heart surgery: a randomized controlled trial. J Thorac Cardiovasc Surg 2011; 142: 148-154.

31. Luo W, Zhu M, Huang R, Zhang Y. A comparison of cardiac post-conditioning and remote pre-conditioning in paediatric cardiac surgery. Cardiol Young 2011; 21: 1-5.

32. Cheung MMH, Kharbanda RK, Konstantinov IE, Shimizu M, Frndova H, Li J, Holtby HM, Cox PN, Smallhorn JF, Van Arsdell GS, Redington AN. Randomized controlled trial of the effects of remote ischemic preconditioning on children undergoing cardiac surgery: first clinical application in humans. J Am Coll Cardiol 2006; 47: 2277-2282.

33. Zhou WW, Zeng DB, Chen RW, Liu J, Yang GX, Liu PB, Zhou X. Limb ischemic preconditioning reduces heart and lung injury after an open heart operation in infants. Pediatr Cardiol 2010; 31: 22-29.

34. Peters J. Remote ischaemic preconditioning of the heart: remote questions, remote importance, or remote preconditions? Basic Res Cardiol 2011; 106: 507-509.

35. Takagi H, Umemoto T. Remote ischemic preconditioning for cardiovascular surgery: an updated meta-analysis of randomized trials. Vasc Endovascular Surg 2011; 45: 511-513.

36. Klatte K, Chaitman BR, Theroux P, Gavard JA, Stocke K, Boyce S, Bartels C, Keller B, Jessel A; GUARDIAN Investigators (The GUARD during Ischemia Against Necrosis). Increased mortality after coronary artery bypass graft surgery is associated with increased levels of postoperative creatine kinase-myocardial band isoenzyme release: results from the GUARDIAN trial. J Am Coll Cardiol 2001; 38: 1070-1077. 